\title{
A prospective feasibility study of respiratory-gated proton beam therapy for liver tumors
}

\section{Citation}

Hong, Theodore S., Thomas F. DeLaney, Harvey J. Mamon, Christopher G. Willett, Beow Y. Yeap, Andrzej Niemierko, John A. Wolfgang, et al. 2014. "A Prospective Feasibility Study of Respiratory-Gated Proton Beam Therapy for Liver Tumors." Practical Radiation Oncology 4 (5) (September): 316-322. doi:10.1016/j.prro.2013.10.002.

\section{Published Version}

10.1016/j.pro.2013.10.002

\section{Permanent link}

http://nrs.harvard.edu/urn-3:HUL.InstRepos:32434447

\section{Terms of Use}

This article was downloaded from Harvard University's DASH repository, and is made available under the terms and conditions applicable to Open Access Policy Articles, as set forth at http:// nrs.harvard.edu/urn-3:HUL.InstRepos:dash.current.terms-of-use\#OAP

\section{Share Your Story}

The Harvard community has made this article openly available.

Please share how this access benefits you. Submit a story.

\section{Accessibility}




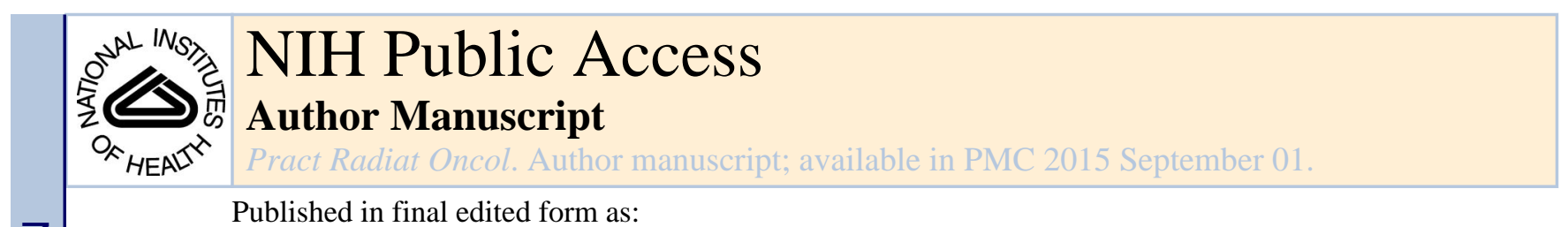

Published in final edited form as:

Pract Radiat Oncol. 2014 ; 4(5): 316-322. doi:10.1016/j.prro.2013.10.002.

\section{A prospective feasibility study of respiratory gated proton beam therapy for liver tumors}

Theodore S. Hong, MD' ${ }^{1}$, Thomas F. DeLaney, MD ${ }^{1}$, Harvey J. Mamon, MD, PhD², Christopher G. Willett, MD ${ }^{3}$, Beow Y. Yeap, ScD ${ }^{5}$, Andrzej Niemierko, PhD ${ }^{1}$, John A. Wolfgang, PhD ${ }^{1}$, Hsiao-Ming Lu, PhD ${ }^{1}$, Judith Adams, CMD ${ }^{1}$, Elizabeth A. Weyman, BA ${ }^{1}$, Ronald S. Arellano, MD ${ }^{4}$, Lawrence S. Blaszkowsky, MD ${ }^{5}$, Jill N. Allen, MD ${ }^{5}$, Kenneth $\mathrm{K}$. Tanabe, $\mathbf{M D}^{6}$, David P. Ryan, $\mathrm{MD}^{5}$, and Andrew X. Zhu, MD, $\mathrm{PhD}^{5}$

${ }^{1}$ Department of Radiation Oncology, Harvard Medical School, Boston, MA.

2Department of Radiation Oncology, Brigham and Women's Hospital/Dana-Farber Cancer Institute, Harvard Medical School, Boston, MA.

${ }^{3}$ Department of Radiation Oncology, Duke University Medical Center, Durham, NC

${ }^{4}$ Department of Radiology, Harvard Medical School, Boston, MA.

${ }^{5}$ Department of Medicine, Harvard Medical School, Boston, MA.

${ }^{6}$ Department of Surgery Massachusetts General Hospital, Harvard Medical School, Boston, MA.

Abstract

Purpose-To evaluate the feasibility of a respiratory-gated proton beam therapy for liver tumors.

Materials and Methods-Fifteen patients were enrolled on a prospective IRB-approved protocol. Eligibility criteria included Childs-Pugh A/B cirrhosis, unresectablebiopsy-proven hepatocellular carcinoma (HCC), intrahepatic cholangiocarcinoma (ICC), or metastatic disease (solid tumors only), 1-3 lesions, and tumor size of $\$ 6 \mathrm{~cm}$. Patients received 15 fractions to a total dose of 45-75 GyE using respiratory-gated proton beam therapy. Gating was performed with an external respiratory position monitoring (RPM) based system.

Results-Of the 15 patients enrolled on this clinical trial, 11 had HCC, 3 had ICC, and 1had metastasis from another primary. Ten patients had a single lesion, 3 patients had 2 lesions, and 2 patients 3 lesions. Toxicities were: Gr 3 bilirubinemia- 2, Gr 3 gastrointestinal bleed- 1, and Gr 5 stomach perforation-1. One patient had a marginal recurrence, 3 had hepatic recurrences elsewhere in the liver, and 2 had extrahepatic recurrence. With a median follow-up for survivors of 69 months, 1-yr, 2-yr, 3 -yr OS is $53 \%, 40 \%$, and $33 \%$ respectively. PFS is $40 \%, 33 \%$ and $27 \%$ at 1,2 , and 3 years, respectively.

(C) 2013 American Society for Radiation Oncology. Published by Elsevier Inc. All rights reserved.

Corresponding author: Theodore S. Hong, M.D. Department of Radiation Oncology Massachusetts General Hospital 100 Blossom Street, COX-3 Boston, MA 02114 Telephone: (617)-726-6050/Fax: (617)-726-3603 tshong1@ partners.org.

Publisher's Disclaimer: This is a PDF file of an unedited manuscript that has been accepted for publication. As a service to our customers we are providing this early version of the manuscript. The manuscript will undergo copyediting, typesetting, and review of the resulting proof before it is published in its final citable form. Please note that during the production process errors may be discovered which could affect the content, and all legal disclaimers that apply to the journal pertain. 
Conclusion-Respiratory-gated proton beam therapy for liver tumors is feasible. Phase II studies for primary liver tumors and metastatic tumorsare underway.

\section{INTRODUCTION}

Ablative therapies for liver tumors offer the potential for long term survivors in select patients with primary and metastatic liver tumors. Radiation therapy for liver tumors has historically been limited by the risk of radiation-induced liver disease (RILD), limiting the use of radiation in the treatment of liver tumors (1). More recently, the development of advanced radiotherapy techniques, like stereotactic body radiotherapy (SBRT) and particle therapy, has re-opened the interest in liver-directed radiation therapy $(2,3)$.

Because of the significant volume effect on the risk of RILD, protons theoretically offer a potential benefit due to the lack of exit dose (4). However, because proton range is so highly dependent on tissue density, treating moving targets can lead to overshooting through normal tissues or underdosing tumor targets. Particularly for liver tumors, where there is an interface of air and soft tissue near the dome of the diaphragm, this risk can be further heightened. Furthermore, because of the greater mechanical complexity of a cyclotron, respiratory gating strategies are more complicated to implement. In a previous study conducted at our institution, a delay of $130 \mathrm{msec}$ was measured in triggering the beam on and off within the gating window (5).

In this pilot study, we evaluate the feasibility of a respiratory gated proton beam therapy in liver tumors.

\section{MATERIAL AND METHODS}

Patients were enrolled on to a prospective institutional review board clinical trial (NCT00465023). Adult patients 18 years or older were required to have biopsy proven unresectable or locally recurrent hepatocellular cancer (HCC), intrahepatic cholangiocarcinoma (ICC), or hepatic metastases. Single or multinodular tumors (up to 3) were permitted, with a maximum size of $6 \mathrm{~cm}$. Patients were required to have no evidence of extrahepatic tumor by CT scan. Karnofsky performance status of 70-100. In patients with underlying cirrhosis, only Child's classification A or B were permitted. No prior liver radiation, including radio-embolization, was permitted. Patients were deemed unresectable after review with transplant surgery and surgical oncology at the institutional multidisciplinary liver conference.

\section{Simulation}

Prior to simulation, fiducials were implanted by interventional radiology, typically superiorly, inferiorly, medially, and laterally, anatomy permitting. The fiducials are gold markers (Part \#351-1, Best Medical, Springfield VA), measuring 0.0325 inches in diameter, and 0.1195 inches in length. The patients then underwent 4-dimensional simulation with intravenous contrast. Respiratory phase, used for later CT reconstruction of 4D motion, was recorded during the cine scan using the Real-time Position Management system (RPM, Varian Medical Systems, Palo Alto, CA). Targets were contoured on the $50 \%$ (end-exhale) 
phase of the 4D CT. A clinical target volume (CTV) expansion of $0-\mathrm{cm} \mathrm{-1} \mathrm{cm}$ was used at the discretion of the treating physician. The precise CTV varied based on the confidence of the treating physician to identify the borders of the lesion on imaging. For the metastatic patient, no CTV expansion was used. For HCC and cholangiocarcinoma, $0.7 \mathrm{~cm}$ was most commonly used. The planning target volume (PTV) margin was customized based on the amount of motion visualized in the gating window $(40 \%-60 \%)$. The treatment fields were planned to PTV with the aperture margin of $10-12 \mathrm{~mm}$, in consideration of the beam penumbra for the required beam energy and the static patient setup uncertainty of our beam/ imaging system. The smearing radius was $5 \mathrm{~mm}$ plus one half of the residual tumor motion over the gated window based on the 4D CT scan.Mean residual tumor motion within the prescribed 30\% gating window, as observed from the 4DCT planning scan, was $5 \mathrm{~mm}$. No abdominal compression or other rigid immobilization was used. No mechanical displacement of mucosal structures from the liver was used.

\section{Dose prescription and normal tissue constraints}

The patients were planned to receive between $45-75 \mathrm{GyE}$ in 15 fractions based on what was achievable given normal tissue constraints (Figure 1). Greater than $90 \%$ of the PTV had to receive the prescription dose. Dose-painting was not permitted. The spinal cord maximum dose allowed was $30 \mathrm{~Gy}$. The stomach maximum dose was originally set at $45 \mathrm{GyE}$ but was reduced to 42 GyE. Other bowel structures, including duodenum, small bowel and large bowel, were restricted to a maximum dose of $45 \mathrm{GyE}$. No more than $2 \mathrm{cc}$ 's of the chest wall, defined as the overlying ribs and intercostal muscles at the level of liver contoured from the costovertebral junction to the sternum or the furthest left extent of the liver, could receive over 60 GyE. The liver-GTV equivalent uniform dose (EUD) could not exceed $20 \mathrm{~Gy}$. If a plan of $45 \mathrm{GyE}$ was not achievable, the patient was taken off study.

\section{Treatment}

All treatments were delivered using $240 \mathrm{MeV}$ protons generated from a cyclotron. Proton beam therapy was delivered using 3D passively scattered protons. Most commonly, 2 fields were used (Figure 1). Respiratory gating was performedusing the RPM system (described above), configured for a $30 \%$ beam-on duty cycle centered on end-of-exhale (50\% phase), corresponding to the physician defined anatomy from the CT planning simulation. Gated orthogonal radiographs (anterior/posterior, right/lateral), manually triggered using the observed RPM respiratory phase signal, were acquired at end-of-exhale and used to confirm the patient treatment position with corresponding endof-exhale DRR's from planning CT. Motion margins describing the range variance of the implanted fiducials, derived from the original 4DCT planning scan, were used to verify the setup position in the presence of respiratory motion. Patients did not receive any breathing coaching.

\section{Follow up}

Patients had follow up visits every three months with CT scans every six months for the first three years. For years 4-5, patients had follow up visits every six months, with yearly CT scans. Toxicity was scored using the common toxicity criteria (CTC) version 3.0. 


\section{Statistics}

Overall survival (OS) as well as the times to local progression and to metastatic disease was calculated starting from the first day of radiation. The OS time of a patient still alive at the time of last follow-up was censored. Progression-free survival (PFS) was measured until a patient had a recurrence documented or died, whichever event was earlier, or otherwise was censored at the date of last follow-up. Survival rates were estimated by the Kaplan-Meier method.

\section{RESULTS}

Of the 15 patients accrued from February 2006 - March 2009, 11 patients had HCC, 3 had ICC and 1 had a liver metastasis from pancreatic adenocarcinoma. Ten patients had 1 lesion, 3 patients had two lesions, and 2 patients had 3 lesions. Nine patients had Child's A cirrhosis and six patients had Child's B cirrhosis. Patient characteristics are listed in Table 1. The median liver size was $1508 \mathrm{cc}$ range (849-2337) cc.

\section{Radiation Dosing}

The dose of radiation received by the patients is listed in Table 2. The median dose was 60 Gy (in 15 fractions) (range (45-75 Gy)

\section{Toxicity}

4/15 patients developed grade 3 or greater toxicity, with some patients developing more than one grade 3 toxicity (Table 3 ). Hyperpigmentation in the radiation portal was the most frequently observed toxicity. Two patients with Childs B cirrhosis developed grade 3 hyperbilirubinemiawhich resolved with conservative management. 1 patient developed a grade 3 bleed in the colon which resolved without surgery. 1 patient developed a grade 5 stomach perforation. There were no cases of radiation induce liver disease (RILD).

\section{Disease-specific outcomes}

There were no in-field local recurrences. 1 patient had a marginal recurrence, 3 had hepatic recurrences elsewhere in the liver, and 2 had extrahepatic recurrence. The patient with a marginal recurrence had tumor in the left lobe of the liver abutting the stomach, with underdosing of the tumor to protect the stomach.

Median survival in all patients was 12 months. With a median follow-up for survivors of 69 months, 1-yr, 2-yr, 3-yr OS is 53\%, 40\%, and 33\% respectively (Figure 2A). Median PFS among all 15 patients was 11 months. PFS is $40 \%, 33 \%$ and $27 \%$ at 1,2 and 3 years, respectively (Figure 2B).

Of the fifteen patients, 4 patients are confirmed alive with over four years of continuous, active follow up. 3 patients had HCC, one patient had IHC. All 4 patients had a solitary tumor. 2 patients received $52.5 \mathrm{~Gy}$ and 1 patient received $60 \mathrm{~Gy}$ and 1 patient received 67.5 Gy. One of the patients with HCC had successful liver transplantation. Another patient with HCC is still alive at 16 months following a marginal recurrence. No patient with multifocal disease is still alive.

Pract Radiat Oncol. Author manuscript; available in PMC 2015 September 01. 


\section{DISCUSSION}

The role of radiation therapy for liver tumors is currently evolving. Prospective studies have shown that photon based radiation therapy is effective in achieving high degrees of local control in both HCC (3) and liver metastases from other primary sites (2).

Protons are an appealing modality for liver tumors due to the lack of exit dose. The primary benefit of the lack of exit dose is that the volume of liver irradiated with protons is less than with photons (4). Given the low functional reserve that many patients with primary liver tumors have due to the underlying risk factor of cirrhosis, protons may represent a safer modality in the ablative treatment of liver tumors. However, protons, because of their greater dependence on tissue density, are more prone to dose perturbations related to organ motions. This uncertainty can lead to underdosing tumors or overdosing normal structures, such as the heart or esophagus, distal to the target. This uncertainty is most significant for lesions at the dome of the liver where organ motion is the greatest. Further complicating the treatment of dome lesions with protons is difference in density of lung versus the liver. At the superior aspect of the target volume, where there is more lung in the field, the protons can range distal to the target towards the heart. This effect is more pronounces with protons in comparison to photons. With respiratory gating, the density of the tissue that the beam encounters is more stable and produces a more stable dosimetric distribution (Figure 4). By restricting the "beam on" time to $40 \%-50 \%$ gating window rather than the $0 \%-50 \%$ ungated window, the dose distribution throughout the treatment remains more consistent. However, the implementation of respiratory gating as a strategy to manage organ motion is a complex problem. In a preclinical study, we observed that a delay of $130 \mathrm{~ms}$ was seen between the gating window and the beam on/off time (5). Because the clinical significance of this was unknown, and also because of the limited experience with treating liver tumors with protons in the western hemisphere, we initiated a pilot study to demonstrate the feasibility of gating liver tumors when delivering high dose proton radiation.

Our clinical results are concordant with the existing literature suggesting that excellent local control is achievable with proton beam therapy. The 15 fraction schedule was selected as the Asian proton hepatoma experience has generally used 10 to 20 fractions (5-14), as opposed to the five fraction stereotactic body radiotherapy (SBRT) schedules. While some of the studies state respiratory gating was implemented, the technical specifics by which the gating was implemented was not described in detail (9-12). The clinical outcomes in these studies have demonstrated impressive local control, with 5 year local control rates exceeding $80 \%$. However, as in this current series, 5 yr overall survival is generally well below $50 \%$, due to the competing risk of poor underlying medical condition and elsewhere recurrences.

However, in this high risk group of unresectable patients, it is compelling that 4/15 patients are confirmed long term survivors beyond 4 years of follow up, including in three patients without transplantation. This suggests that long term survival is possible in appropriately selected unresectable patients.

In this study, the liver was often not the dose limiting structure. Instead, prescription dose was more frequently limited by mucosal structures such as the stomach and colon. Because the protocol did not allow for differential dosing, the dose to the tumor was often de- 
escalated to 45 Gy to meet the mucosal dose constraint. In spite of this, two of the fifteen patients experienced mucosal injury, one of which was self-limited, and the other resulted in death. The patient who died from stomach perforation had a 45 Gy point dose in the lesser curvature of his stomach, which was consistent with the protocol constraints. He also had complex underlying pathophysiology as he was born with a single ventricle and had developed cirrhosis secondary to viral hepatitis acquired from multiple blood transfusions that were given to him when he was a child. His portal hypertension due to cirrhosis and his clinical right-sided heart failure were felt to contribute to his lack of reserve and inability to recover from the attempt to operatively control his bleeding. After this event, the maximum dose constraint to the stomach was subsequently lowered to $42 \mathrm{~Gy}$. This problem of mucosal tolerance has led us to use manual displacement with alloderm to separate mucosal structures from bowel (15). Additionally, to address potential range uncertainty and hot spots in mucosal structures, we have since adopted to two-day simulation process to study potential interfractional changes in respiratory amplitude and in the location of the at-risk mucosal structures.

The limitations of this study are its small size and heterogeneous inclusion criteria, making it difficult to draw any conclusions about efficacy in a uniform group of patients. Accordingly we are accruing to separate phase II studies evaluating proton beam therapy in patients with primary liver tumors and liver metastases. The phase II study in primary liver tumors includes patients with hepatic-confined, unresectable HCC and ICC. This multi-institutional study uses doses to $67.5 \mathrm{~Gy}$ for peripheral tumors and $58.05 \mathrm{~Gy}$ for central tumors defined as within $2 \mathrm{~cm}$ of the portahepatis. Unlike this current study, underdosing of the PTV is permitted to as low as $45 \mathrm{~Gy}$. The primary endpoint of this 90 patient study is 2-year local control exceeding $85 \%$. The phase II study of patients with liver metastases uses a 5-fraction individualized dosing strategy, with a primary endpoint of 1 year local control exceeding $80 \%$. The study is planned to accrue 110 patients. This current feasibility studied altered our clinical practice in how we protect mucosal surfaces and provided the foundation for formal evaluation in specific disease cohorts.

\section{Acknowledgments}

This study was supported by NIH: 2P01CA021239-29A1 REVISED

\section{References}

1. Dawson LA, McGinn CJ, Normolle D, et al. Escalated focal liver radiation and concurrent hepatic artery fluorodeoxyuridine for unresectable intrahepatic malignancies. J ClinOncol. 2000; 18:2210 2218.

2. Lee MT, Kim JJ, Diniwell R, et al. Phase I study of individualized stereotactic body radiotherapy of liver metastases. J ClinOncol. 2009; 27:1585-91.

3. Bujold A, Massey CA, Kim JJ, et al. Sequential phase I and II trials of stereotactic body radiotherapy for locally advanced hepatocellular carcinoma. J ClinOncol. 2013; 31:1631-9.

4. Wang X, Krishnan S, Zhang X, et al. Proton radiotherapy for liver tumors: dosimetric advantages over photon plans. Med Dosim. 2008; 33:259-267. [PubMed: 18973852]

5. Lu HM, Brett R, Sharp G, et al. A respiratory-gated treatment system for proton therapy. Med Phys. 2007; 34:3273-8. [PubMed: 17879790] 
6. Hata M, Tokuuye K, Sugahara S, et al. Proton beam therapy for hepatocellular carcinoma with limited treatment options. Cancer. 2006; 107:591-598. [PubMed: 16804931]

7. Hata M, Tokuuye K, Sugahara S, et al. Proton beam therapy for hepatocellular carcinoma with portal vein tumor thrombus. Cancer. 2005; 104:794-801. [PubMed: 15981284]

8. Hata M, Tokuuye K, Sugahara S, et al. Proton beam therapy for aged patients with hepatocellular carcinoma. Int J RadiatOncolBiolPhys. 2007; 69:805-812.

9. Mizumoto M, Tokuuye K, Sugahara S, et al. Proton beam therapy for hepatocellular carcinoma adjacent to the portahepatis. Int J RadiatOncolBiolPhys. 2008; 71:462-467.

10. Sugahara S, Oshiro Y, Nakayama H, et al. Proton beam therapy for large hepatocellular carcinoma. Int J RadiatOncolBiolPhys. 2010; 76:460-466.

11. Nakayama H, Sugahara S, Tokita M, et al. Proton beam therapy for hepatocellular carcinoma: the University of Tsukuba experience. Cancer. 2009; 115:5499-5506. [PubMed: 19645024]

12. Fukumitsu N, Sugahara S, Nakayama H, et al. A prospective study of hypofractionated proton beam therapy for patients with hepatocellular carcinoma. Int J Radiat Oncol Biol Phys. 2009; 74:831-836. [PubMed: 19304408]

13. Hashimoto T, Tokuuye K, Fukumitsu N, et al. Repeated proton beam therapy for hepatocellular carcinoma. Int J Radiat Oncol Biol Phys. 2006; 65:196-202. [PubMed: 16563656]

14. Kawashima M, Furuse J, Nishio T, et al. Phase II study of radiotherapy employing proton beam for hepatocellular carcinoma. J Clin Oncol. 2005; 23:1839-1846. [PubMed: 15774777]

15. Yoon SS, Aloia TA, Haynes AB, et al. Surgical placement of biological mesh spacers to displace bowel away from unresectable liver tumors followed by delivery of dose-intense radiation therapy. Pract Radiat Oncol. 2013 (in press). 
Proton Plan
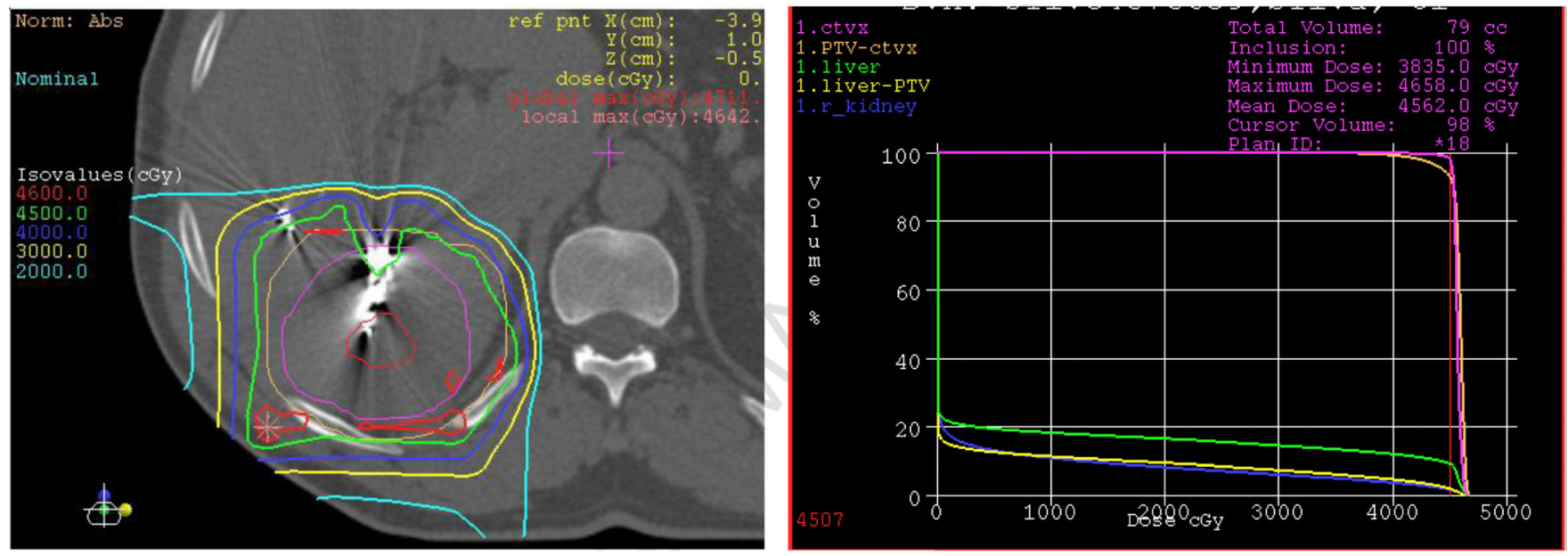

Figure 1.

Proton plan for patient with intrahepatic cholangiocarcinoma. 

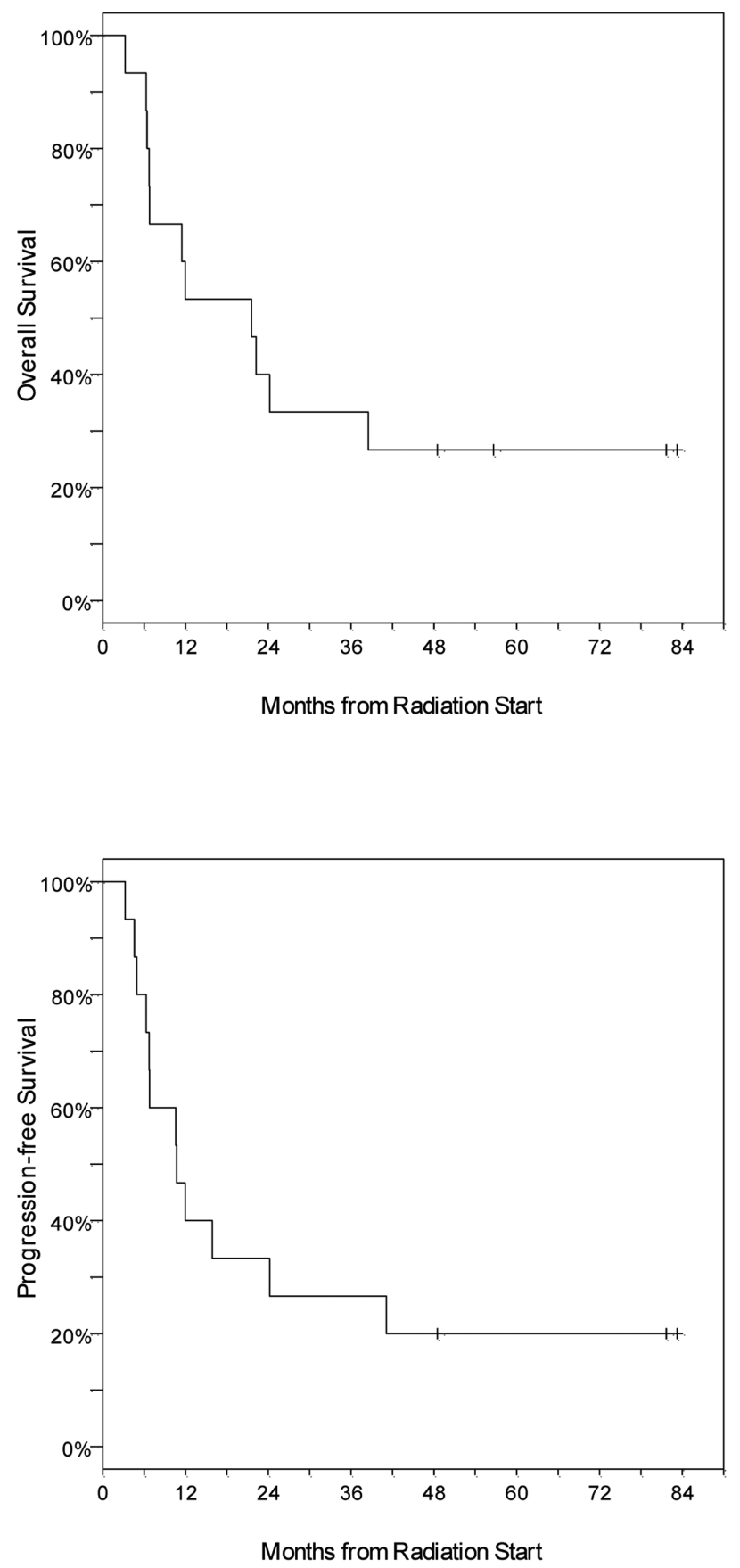

Figure 2.

A: Overall Survival. B: Progression-free Survival. 

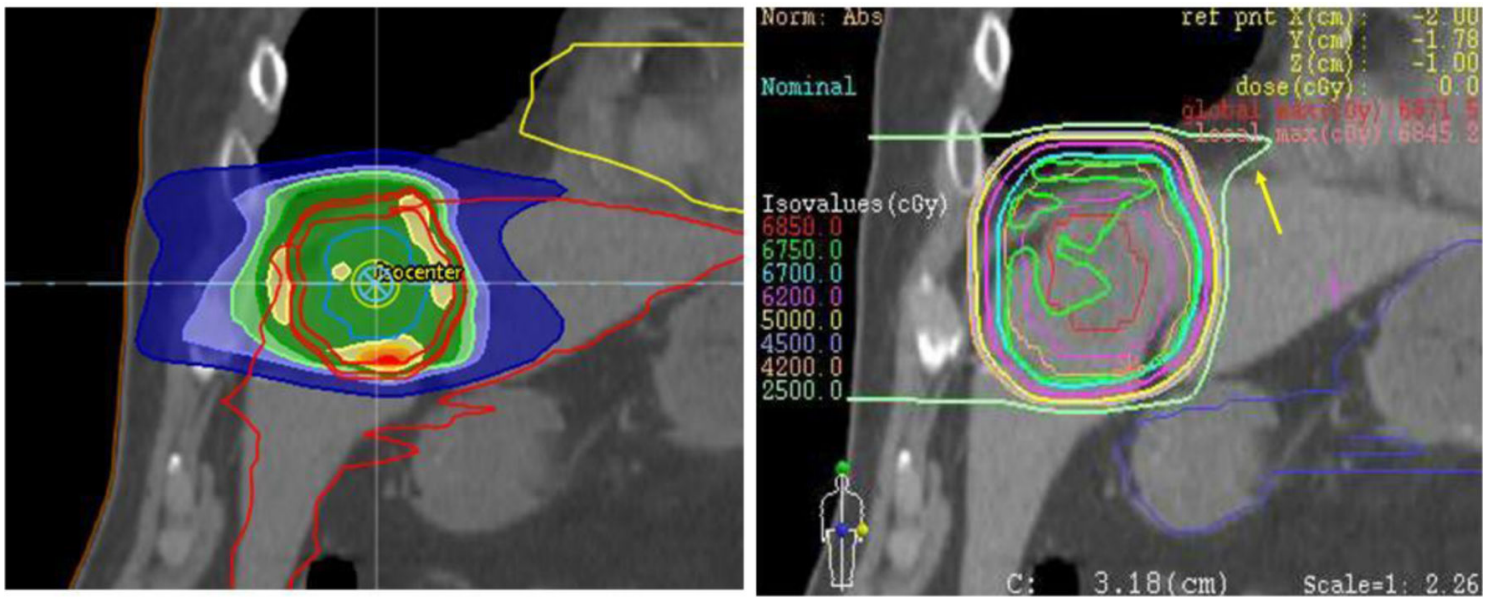

Figure 3.

Photon vs. proton treatment plan for a dome lesion. The proton plan (left) shows the 25 Gy at the superior aspect of the field ranging out towards the heart due to the large amount of lung in the field. 
$0 \%$

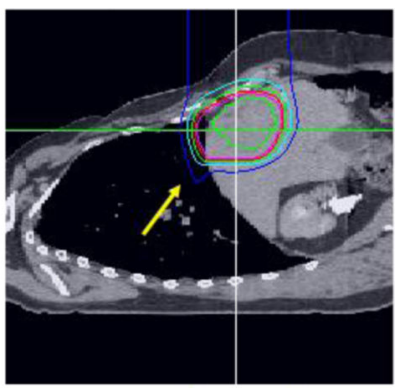

$30 \%$

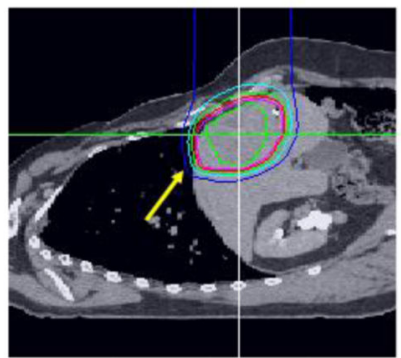

$50 \%$

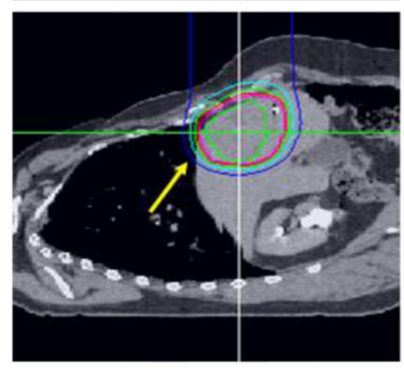

$40 \%$

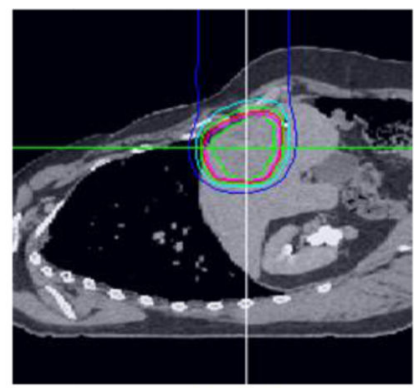

$45 \%$

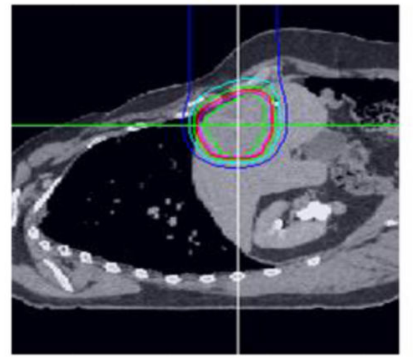

$50 \%$

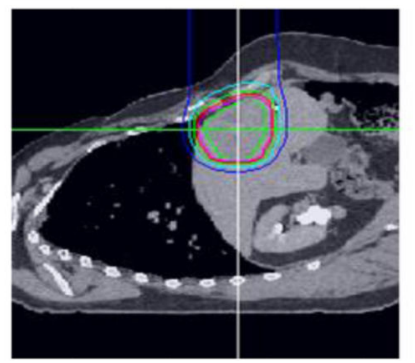

Figure 4.

Ungated vs. gated proton treatment. On the left, the isodose distribution at the $0 \%, 30 \%$, and $50 \%$ respiratory phases are shown. The field is noticeably larger and the dose distribution above the diaphragm varies with phase. In contrast, in the gated image, the isodose distributions do not perceptibly change through the gating window. 
Table1 Baseline Characteristics

\begin{tabular}{lc}
\hline Characteristic & \\
\hline Age at Radiation & \\
\hline Median (range) & 58 years (25-83) \\
Gender & $12(80 \%)$ \\
Male & $3(20 \%)$ \\
Female & \\
Childs-Pugh Score & $3(60 \%)$ \\
A & $1(40 \%)$ \\
B & \\
Tumor Type & $11(73 \%)$ \\
HCC & $3(20 \%)$ \\
ICC & $1(7 \%)$ \\
Metastatic & \\
Number lesions & $10(67 \%)$ \\
1 & $3(20 \%)$ \\
2 & $2(13 \%)$ \\
3 & $1508 \mathrm{cc}(849-2337)$ \\
Whole Liver Volume & \\
Median (range) & \\
CTV (cc) & \\
Median (range) & $124.03(20.1-581)$
\end{tabular}

Pract Radiat Oncol. Author manuscript; available in PMC 2015 September 01. 


\section{Table 2}

Radiation Dosing

\begin{tabular}{ccc}
\hline Dose & Number of Patients & Number of Lesions \\
\hline 45 Gy & 3 & 4 \\
$52.5 \mathrm{~Gy}$ & 4 & 5 \\
$60 \mathrm{~Gy}$ & 3 & 4 \\
$67.5 \mathrm{~Gy}$ & 3 & 5 \\
$75 \mathrm{~Gy}$ & 2 & 4
\end{tabular}


Table 3

Related Toxicity

\begin{tabular}{|r|c|c|c|c|c|}
\hline Toxicity Description & Grade 1 & Grade 2 & Grade 3 & Grade 4 & Grade 5 \\
\hline Acute & & & & & \\
\hline Thrombocytopenia & 0 & 1 & 3 & 0 & 0 \\
\hline Hyperbilirubinemia & 0 & 4 & 1 & 0 & 0 \\
\hline Elevated Transaminases & 0 & 1 & 0 & 0 & 0 \\
\hline Late & & & & & \\
\hline Hyperpigmentation of skin & 5 & 1 & 0 & 0 & 0 \\
\hline Gastrointestinal Bleed & 0 & 0 & 1 & 0 & 0 \\
\hline Gastrointestinal Perforation & 0 & 0 & 0 & 0 & 1 \\
\hline Total & 5 & 7 & 7 & 0 & 1 \\
\hline Total patients with toxicity & 5 & 5 & 4 & 0 & 1 \\
\hline
\end{tabular}

